\title{
Countable products of probabilistic normed spaces
}

\author{
Bernardo Lafuerza-Guillén ${ }^{\mathrm{a}}$, Carlo Sempi ${ }^{\mathrm{b}, *}$, Gaoxun Zhang ${ }^{\mathrm{c}}$, Minxian Zhang ${ }^{\mathrm{d}}$ \\ a Departamento de Estadística y Matemática Aplicada, Universidad de Almería, Almería, 04120. Spain \\ b Dipartimento di Matematica, "Ennio De Giorgi", Università del Salento, Lecce, 73100, Italy \\ ${ }^{c}$ Tian Fu College of Southwestern University of Finance and Economics, Mianyang, Sichuan 621000, PR China \\ ${ }^{d}$ Department of Computation Science, Chengdu University of Information Technology, Chengdu, Sichuan 610225, PR China
}

\section{A R T I C L E I N F O}

\section{Article history:}

Received 23 January 2009

Accepted 26 February 2009

\section{MSC:}

$54 \mathrm{E} 70$

46570

Keywords:

Probabilistic normed spaces

Countable product

Triangle function

\section{A B S T R A C T}

Countable products of probabilistic normed spaces are introduced and studied. In particular, a comparison is made with the analogous constructions for probabilistic metric spaces.

(C) 2009 Published by Elsevier Ltd

\section{Introduction}

Probabilistic Normed (briefly PN) spaces were introduced by Šerstnev in a series of papers [1-4]. Then a new definition was proposed by Alsina, Schweizer and Sklar [5]. This new definition revived the study of PN spaces. The properties of these spaces were studied by several authors; here we shall mention [6-9] (but see also the survey paper [10]). We recall below for the reader's convenience the definition of a PN space; the notation is essentially that fixed by the classic book by Schweizer and Sklar [11].

Definition 1.1. A probabilistic normed space is a quadruple $\left(V, \nu, \tau, \tau^{*}\right)$, where $V$ is a real linear space, $\tau$ and $\tau^{*}$ are continuous triangle functions and the mapping $v: V \rightarrow \Delta^{+}$satisfies, for all $p$ and $q$ in $V$, the conditions

(N1) $v_{p}=\epsilon_{0}$ if, and only if, $p=\theta(\theta$ is the null vector in $V)$;

(N2) $\forall p \in V \quad v_{-p}=v_{p}$;

(N3) $v_{p+q} \geq \tau\left(v_{p}, v_{q}\right)$;

(N4) $\forall \alpha \in[0,1] \quad v_{p} \leq \tau^{*}\left(v_{\alpha p}, v_{(1-\alpha) p}\right)$.

The function $v$ is called the probabilistic norm. If $\left(V, v, \tau, \tau^{*}\right)$ satisfies the condition, weaker than (N1),

$$
v_{\theta}=\epsilon_{0},
$$

then it is called a Probabilistic Pseudo-Normed space (briefly, a PPN space). If $\tau=\tau_{T}$ and $\tau^{*}=\tau_{T^{*}}$ for some continuous $t$-norm $T$ and its $t$-conorm $T^{*}$ then $\left(V, v, \tau_{T}, \tau_{T^{*}}\right)$ is denoted by $(V, v, T)$ and is a Menger PN space.

\footnotetext{
* Corresponding author. Tel.: +39 0832351786; fax: +39 0832297410.

E-mail addresses: blafuerz@ual.es (B. Lafuerza-Guillén), carlo.sempi@unisalento.it (C. Sempi), zhanggaoxun002@163.com (G. Zhang), mxzhang@cuit.edu.cn (M. Zhang).
}

0362-546X/\$ - see front matter (c) 2009 Published by Elsevier Ltd doi: $10.1016 / \mathrm{j} . n \mathrm{n} .2009 .02 .124$ 
A PN space is called a Šerstnev space if it satisfies conditions (N1), (N3) and following condition

(S) $\forall \alpha \in \mathbb{R} \backslash\{0\} \forall x \in \overline{\mathbb{R}}_{+} \quad v_{\alpha p}(x)=v_{p}\left(\frac{x}{|\alpha|}\right)$,

which implies (N2) and (N4) in the strengthened form

$$
\forall \alpha \in[0,1] \quad \forall p \in V \quad v_{\alpha p}=\tau_{M}\left(v_{\alpha p}, v_{(1-\alpha) p}\right) .
$$

The definition of a $t$-norm may be found in [11-13].

Some examples of $t$-norms $T$ and its $t$-conorms $T^{*}$ are: $M(x, y)=\min \{x, y\}, \Pi(x, y)=x \cdot y$ and $M^{*}(x, y)=\max \{x, y\}$ $\Pi^{*}(x, y)=x+y-x \cdot y$. We recall the following sequence of inequalities:

$$
T \leq M<M^{*} \leq T^{*},
$$

and in particular

$$
W<\Pi<M<M^{*}<\Pi^{*}<W^{*} .
$$

Definition 1.2. A triangle function is a mapping $\tau$ from $\Delta^{+} \times \Delta^{+}$into $\Delta^{+}$such that, for all $F, G, H, K$ in $\Delta^{+}$,

(1) $\tau\left(F, \epsilon_{0}\right)=F$

(2) $\tau(F, G)=\tau(G, F)$,

(3) $\tau(F, G) \leq \tau(H, K)$ whenever $F \leq H, G \leq K$,

(4) $\tau(\tau(F, G), H)=\tau(F, \tau(G, H))$.

Relevant triangle functions are the functions $\tau_{T}, \tau_{T^{*}}$ and those of the form $\Pi_{T}$ which, for any continuous $t$-norm $T$, and any $x>0$, are given by

$$
\begin{aligned}
& \tau_{T}(F, G)(x)=\sup \{T(F(u), G(v)) \mid u+v=x\}, \\
& \tau_{T^{*}}(F, G)(x)=\inf \left\{T^{*}(F(u), G(v)) \mid u+v=x\right\}
\end{aligned}
$$

and

$$
\Pi_{T}(F, G)(x)=T(F(x), G(x)) .
$$

See $[14,15]$ for a recent study of triangle functions.

The purpose of this note is to study the countable product of PN spaces. The case of the finite product of PN spaces was studied by the first author in [16]. The countable product of probabilistic metric spaces was the object of the two importan papers by Alsina [17] and Alsina and Schweizer [18]; the differences between these works and the present one will be noted; in the first place, while a single triangle function appears in the definition of a probabilistic metric space, two triangle functions, $\tau$ and $\tau^{*}$, have to be considered in a PN space.

\section{The dominance relation}

Definition 2.1. Let $(S, \leq)$ be a partially ordered set and let $f$ and $g$ be commutative and associative binary operations on $S$ with common identity $e$. Then $f$ dominates $g$, and one writes $f \gg g$, if, for all $x_{1}, x_{2}, y_{1}, y_{2}$ in $S$,

$$
f\left(g\left(x_{1}, y_{1}\right), g\left(x_{2}, y_{2}\right)\right) \geq g\left(f\left(x_{1}, x_{2}\right), f\left(y_{1}, y_{2}\right)\right) \text {. }
$$

Setting $y_{1}=x_{2}=e$ in this inequality, one has $f\left(x_{1}, y_{2}\right) \geq g\left(x_{1}, y_{2}\right)$; thus $f \gg g$ implies $f \geq g$, which in turn implies that the dominance relation is antisymmetric. It is easily shown that the dominance relation is also reflexive. However, althougl it is known that the dominance relation is transitive on certain subsets of the set of continuous $t$-norms (see [19-21]), it is not, in general, transitive, as examples due to Sherwood [13] and Sarkoci [22] show.

We are interested in the dominance relation as it applies to $t$-norms, $s$-norms ${ }^{1}$ and triangle functions. Here the following are known:

Lemma 2.1. The following statements hold:

(a) For every $t$-norm $T, M \gg T$;

(b) For every s-norm $S, S \gg M^{*}$;

(c) For every continuous $t$-norm $T, \Pi_{T} \gg \tau_{T}$;

(d) For every triangle function $\tau, \Pi_{M} \gg \tau$.

\footnotetext{
$1 s$-norms are often called $t$-conorms in the literature.
} 
Proof. (a) For all $x_{1}, x_{2}, y_{1}$ and $y_{2}$ in $I$, one has

$$
x_{j} \geq M\left(x_{1}, x_{2}\right), \quad y_{j} \geq M\left(y_{1}, y_{2}\right) \quad(j=1,2) ;
$$

thus

$$
\begin{aligned}
& T\left(x_{1}, y_{1}\right) \geq T\left(M\left(x_{1}, x_{2}\right), M\left(y_{1}, y_{2}\right)\right), \\
& T\left(x_{2}, y_{2}\right) \geq T\left(M\left(x_{1}, x_{2}\right), M\left(y_{1}, y_{2}\right)\right),
\end{aligned}
$$

whence

$M\left(T\left(x_{1}, y_{1}\right), T\left(x_{2}, y_{2}\right)\right) \geq T\left(M\left(x_{1}, x_{2}\right), M\left(y_{1}, y_{2}\right)\right)$,

i.e. $M \gg T$. Corresponding arguments prove (b), (c) and (d).

Next, a straightforward calculation yields

Lemma 2.2. If $T_{1}$ and $T_{2}$ are $t$-norms such that $T_{1} \gg T_{2}$, then $T_{2}^{*} \gg T_{1}^{*}$, and conversely.

The following theorem is due to Tardiff [23].

Theorem 2.1. The following statements are equivalent for all continuous $t$-norms $T_{1}$ and $T_{2}$ :
(1) $T_{1} \gg T_{2}$;
(2) $\Pi_{T_{1}} \gg \Pi_{T_{2}}$;
(3) $\tau_{T_{1}} \gg \tau_{T_{2}}$;
(4) $\Pi_{T_{1}} \gg \tau_{T_{2}}$;
(5) $\tau_{T_{2}^{*}} \gg \tau_{T_{1}^{*}}$.

\section{Main results: (I)-The $\Sigma$-product}

In a given PN space $\left(V, \nu, \tau, \tau^{*}\right)$ the value of the probabilistic norm of $p \in V$ at the point $t$ will be denoted either by $\nu(p)(t)$ or by $v_{p}(t)$.

Definition 3.1. Given a sequence $\left(V_{j}, v_{j}, \tau_{j}, \tau_{j}^{*}\right)_{j \in \mathrm{N}}$ of PN spaces and a sequence $\left(\beta_{j}\right)_{j \in \mathrm{N}}$ of strictly positive real numbers such that $\sum_{j \in \mathbf{N}} \beta_{j}=1$, their $\Sigma$-product is the pair $(V, \nu)$ where $V$ is the countable cartesian product of the spaces $V_{j}, V:=\prod_{j \in \mathbb{N}} V_{j}$, and where, for $p=\left(p_{j}\right) \in V$,

$$
v_{p}:=\sum_{j \in \mathbf{N}} \beta_{j} v_{j}\left(p_{j}\right)
$$

Our first result is the following.

Theorem 3.1. Let $\left(V_{j}, v_{j}, \tau_{j}, \tau_{j}^{*}\right)_{j \in \mathbb{N}}$ be a sequence of PN spaces such that for, every $j \in \mathbf{N}, \tau_{j} \geq \tau_{W}$ and $\tau_{j}^{*} \leq \tau_{W^{*}}$. Then their $\Sigma$-product $(V, v)$ is a Menger PN space under $W$.

Proof. Let $\theta:=\left(\theta_{1}, \theta_{2}, \ldots, \theta_{j}, \ldots\right)$, where $\theta_{j}$ is the origin of the vector space $V_{j}(j \in \mathbf{N})$. Then

$$
v_{\theta}=\sum_{j \in \mathbf{N}} \beta_{j} v_{j}\left(\theta_{j}\right)=\sum_{j \in \mathbf{N}} \beta_{j} \epsilon_{0}=\epsilon_{0} .
$$

In the other direction, if $v_{p}=\epsilon_{0}$, then one has $\nu_{p}(t)=1$ for every $t>0$; thus

$$
1=\sum_{j \in \mathbf{N}} \beta_{j} v_{j}\left(p_{j}\right)(t) \leq \sum_{j \in \mathbf{N}} \beta_{j}=1
$$

so that one has, for every $j \in \mathbf{N}$ and every $t>0, v_{j}\left(p_{j}\right)(t)=1$, namely $p_{j}=\theta_{j}$ and, therefore, $p=\theta$. This proves (N1). The proof of (N2) is trivial. As for (N3), one has, for every $t>0$,

$$
\begin{aligned}
v_{p+q}(t) & =\sum_{j \in \mathbf{N}} \beta_{j} v_{j}\left(p_{j}+q_{j}\right)(t) \geq \sum_{j \in \mathbb{N}} \beta_{j} \tau_{j}\left(v_{j}\left(p_{j}\right), v_{j}\left(q_{j}\right)\right)(t) \\
& \geq \sum_{j \in \mathbf{N}} \beta_{j} \tau_{W}\left(v_{j}\left(p_{j}\right), v_{j}\left(q_{j}\right)\right)(t) \\
& =\sum_{j \in \mathbb{N}} \beta_{j} \sup _{u \in[0, t]} W\left(v_{j}\left(p_{j}\right)(u), v_{j}\left(q_{j}\right)(t-u)\right) ;
\end{aligned}
$$


thus, for every $u$ in $[0, t]$,

$$
\begin{aligned}
v_{p+q}(t) & \geq \sum_{j \in \mathbf{N}} \beta_{j} \max \left\{v_{j}\left(p_{j}\right)(u)+v_{j}\left(q_{j}\right)(t-u)-1,0\right\} \\
& \geq \sum_{j \in \mathbf{N}} \beta_{j}\left\{v_{j}\left(p_{j}\right)(u)+v_{j}\left(q_{j}\right)(t-u)-1\right\} \\
& =v_{p}(u)+v_{q}(t-u)-1,
\end{aligned}
$$

and, hence

$$
v_{p+q}(t) \geq \sup _{u \in[0, t]}\left\{v_{p}(u)+v_{q}(t-u)-1\right\} ;
$$

since $v_{p+q} \geq 0$, one has $v_{p+q} \geq \tau_{W}\left(v_{p}, v_{q}\right)$, which proves (N3).

Similarly, for every $\alpha \in[0,1]$ for every $p \in V$ and for every $t>0$, one has

$$
\begin{aligned}
v_{p}(t) & =\sum_{j \in \mathbb{N}} \beta_{j} v_{j}\left(p_{j}\right)(t) \leq \sum_{j \in \mathbb{N}} \beta_{j} \tau_{j}^{*}\left(v_{j}\left(\alpha p_{j}\right), v_{j}\left((1-\alpha) p_{j}\right)\right)(t) \\
& \leq \sum_{j \in \mathbb{N}} \beta_{j} \tau_{W^{*}}\left(v_{j}\left(\alpha p_{j}\right), v_{j}\left((1-\alpha) p_{j}\right)\right)(t) \\
& =\sum_{j \in \mathbb{N}} \beta_{j} \inf _{u \in[0, t]} W^{*}\left(v_{j}\left(\alpha p_{j}\right)(u), v_{j}\left((1-\alpha) p_{j}\right)(t-u)\right) .
\end{aligned}
$$

Recalling that $W^{*}(s, t)=\min \{s+t, 1\}$, one has, for every $u$ in $[0, t]$,

$$
\begin{aligned}
v_{p}(t) & \leq \sum_{j \in \mathbb{N}} \beta_{j} W^{*}\left(v_{j}\left(\alpha p_{j}\right)(u), v_{j}\left((1-\alpha) p_{j}\right)(t-u)\right) \\
& \leq \sum_{j \in \mathbb{N}} \beta_{j}\left(v_{j}\left(\alpha p_{j}\right)(u)+v_{j}\left((1-\alpha) p_{j}\right)(t-u)\right) \\
& =v_{\alpha p}(u)+v_{(1-\alpha) p}(t-u),
\end{aligned}
$$

so that

$$
v_{p}(t) \leq \inf _{u \in[0, t]}\left\{v_{\alpha p}(u)+v_{(1-\alpha) p}(t-u)\right\}
$$

since $v_{p} \leq 1$, one has $v_{p} \leq \tau_{W^{*}}\left(v_{\alpha p}, v_{(1-\alpha) p}\right)$, which establishes (N4) and concludes the proof.

Corollary 3.1. If $\left(V_{j}, v_{j}\right)_{j \in \mathrm{N}}$ is a sequence of Menger PN spaces under $W$, then their $\Sigma$-product is again a Menger PN space under W.

However, even if $\left(V_{j}, v_{j}\right)_{j \in \mathbb{N}}$ is a sequence of PN spaces with respect to the same triangle functions $\tau$ and $\tau^{*}$, their $\Sigma$ product need not be a PN space with respect to the same triangle functions. In order to see this it is sufficient to modify Example 1.3 in Alsina's paper [17]

Example 3.1. Consider the simple space $(\mathbb{R},|\cdot|, U)$ where $U$ is the d.f. of a random variable uniformly distributed on the interval $(0,1)$ and the sequences $p$ and $q$ in $\prod_{n \in \mathbb{N}} \mathbb{R}$ given by

$$
p:=\{c n: n \in \mathbf{N}\} \text { and } q:=\{n: n \in \mathbf{N}\} \text {, }
$$

Assume that $\beta_{1}:=\max \left\{\beta_{j}: j \in \mathbf{N}\right\}$ and, moreover, assume that the constant $\left.c \in\right] 0,1 / 2[$ is small enough to satisfy the inequality

$$
\beta_{1}>\frac{1}{c+1} .
$$

Thus, for every $t>0$,

$$
v_{p+q}(t)=\sum_{j \in \mathbb{N}} \beta_{j} U\left(\frac{t}{(c+1) j}\right)<U\left(\frac{t}{c+1}\right) .
$$

In particular,

$$
v_{p+q}(1)<\frac{1}{c+1}
$$


On the other hand

$$
v_{p}(1 / 2)=\sum_{j \in \mathbf{N}} \beta_{j} U\left(\frac{1}{2 c j}\right)>\beta_{1} U\left(\frac{1}{2 c}\right)=\beta_{1}
$$

since $1 /(2 c)>1$, and

$$
v_{q}(1 / 2)=\sum_{j \in \mathbb{N}} \beta_{j} U\left(\frac{1}{2 j}\right)>\beta_{1} U\left(\frac{1}{c}\right)=\beta_{1},
$$

so that

$$
\tau_{M}\left(v_{p}, v_{q}\right)(1) \geq v_{p}(1 / 2) \wedge v_{q}(1 / 2) \geq \beta_{1} .
$$

Therefore one has, because of Eq. (3.1),

$$
v_{p+q}(1)<\frac{1}{c+1}<\beta_{1} \leq \tau_{M}\left(v_{p}, v_{q}\right)(1),
$$

viz. the $\Sigma$-product of the sequence of simple spaces we have considered is not a simple space.

The previous example also exhibits one drawback of the construction of the $\Sigma$-product of a sequence of PN spaces, namely that even when $\left(V_{j}, \nu_{j}, \tau, \tau^{*}\right)_{j \in N}$ is a sequence of PN spaces under the same triangle functions $\tau$ and $\tau^{*}$, their $\Sigma$-product need not be a PN space under the same two triangle functions. This drawback is evident also in another class of examples, which we now proceed to construct.

Definition 3.2. The pair $(V, v)$ is said to be a Probabilistic Semi-Normed space (briefly a PSN space) when $v: V \rightarrow \Delta^{+}$ satisfies (N1) and (N2). A PSN space $(V, v)$ is said to be a Wald space, when $\tau=*$, namely when the triangle function $\tau$ is the convolution,

$$
v_{p+q} \geq v_{p} * v_{q}
$$

In a PN space with $\tau_{T}=\tau_{\Pi}$, where $\Pi$ is the $t$-norm $\Pi(a, b)=a \cdot b$, one has

$$
v_{p+q}(x+y) \geq v_{p}(x) \cdot v_{q}(y) .
$$

Theorem 3.2. A Wald space $(V, v, *)$ can be made in a PN space $\left(V, v, *, \Pi_{T^{*}}\right)$, for every $t$-norm $T$.

Proof. The inequality $\nu_{p} \leq v_{\beta p}$, which holds for every $p \in V$ and for every $\beta \in[0,1]$, yields, when applied to $\beta=\alpha$ and $\beta=1-\alpha(\alpha \in[0,1])$,

$$
v_{p} \leq \min \left\{v_{\alpha p}, v_{(1-\alpha) p}\right\}=\Pi_{M}\left(v_{\alpha p}, v_{(1-\alpha) p}\right) \leq \Pi_{T^{*}}\left(v_{\alpha p}, v_{(1-\alpha) p}\right) \text {, }
$$

which concludes the proof.

Example 3.2. The quadruple $\left(V, v, *, \Pi_{M}\right)$ where $V$ is a normed linear space and the probabilistic norm $v$ is defined via

$$
v_{p}(x):=\frac{x}{x+1} \mathrm{e}^{-\|p\|}
$$

and $*$ is convolution, is a PN space; it is neither a Šerstnev space nor a topological vector space. In fact, (N1) and (N2) are obvious.

(N3) For every $x>0$ and for all $p$ and $q$ in $V$, one has explicitly, setting $h:=x+1>1$,

$$
\begin{aligned}
\int_{0}^{x} \nu_{p}(x-t) \mathrm{d} v_{q}(t) & =\int_{0}^{x} \frac{x-t}{x-t+1} \mathrm{e}^{-\|p\|} \frac{1}{(t+1)^{2}} \mathrm{e}^{-\|q\|} \mathrm{d} t \\
& =\mathrm{e}^{-(\|p\|+\|q\|)}\left[\int_{0}^{x} \frac{\mathrm{d} t}{(t+1)^{2}}-\int_{0}^{x} \frac{\mathrm{d} t}{(x+1-t)(t+1)^{2}}\right] \\
& =\mathrm{e}^{-(\|p\|+\|q\|)}\left(1-\frac{1}{h}-\frac{2 \ln h}{(1+h)^{2}}-\frac{x}{(1+h) h}\right) .
\end{aligned}
$$


Property (N3) is equivalent, in turn to each of the following inequalities

$$
\begin{aligned}
& \frac{x}{h} \mathrm{e}^{-\|p+q\|} \geq \mathrm{e}^{-(\|p\|+\|q\|)}\left(1-\frac{1}{h}-\frac{2 \ln h}{(1+h)^{2}}-\frac{x}{(1+h) h}\right), \\
& \frac{x}{h} \geq \mathrm{e}^{\|p+q\|-(\|p\|+\|q\|)}\left(1-\frac{1}{h}-\frac{2 \ln h}{(1+h)^{2}}-\frac{x}{(1+h) h}\right), \\
& x \geq \frac{1}{\mathrm{e}^{\|p\|+\|q\|-\|p+q\|}}\left(x-\frac{2 h \ln h}{(1+h)^{2}}-\frac{x}{(1+h) h}\right) \\
& x \geq \frac{1}{\mathrm{e}^{\|p\|+\|q\|-\|p+q\|}}\left(x \frac{h}{1+h}-\frac{2 h \ln h}{(1+h)^{2}}\right) .
\end{aligned}
$$

But

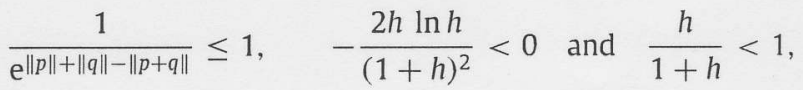

so that

$$
x \geq \frac{1}{\mathrm{e}^{\|p\|+\|q\|-\|p+q\|}} \times \frac{h}{1+h} \geq \frac{1}{\mathrm{e}^{\|p\|+\|q\|-\|p+q\|}}\left(x \frac{h}{1+h}-\frac{2 h \ln h}{(1+h)^{2}}\right),
$$

which proves (N3).

(N4) is immediate since for every $p \in V$ and for every $\alpha \in(0,1)$, one has $\|p\| \geq \alpha\|p\|$ and $\|p\| \geq(1-\alpha)\|p\|$.

Moreover, $\left(V, v, *, \Pi_{M}\right)$ is not a Šerstnev PN space because in this case the equation $(\breve{S})$ is equivalent to the relationship

$$
\frac{x+1}{x+|\alpha|}=\mathrm{e}^{(1-\alpha)\|p\|}
$$

which, for $x \neq-|\alpha|$ is an equation that admits the unique solution

$$
x=\frac{|\alpha| \mathrm{e}^{(1-\alpha)\|p\|}-1}{1-\mathrm{e}^{(1-\alpha)\|p\|}}
$$

this means that equation ( $\breve{S})$ cannot hold for all $x>0$ and $p \in V$.

Example 3.3. The $\Sigma$-product of Wald PN spaces is not necessarily a Wald PN space.

Consider the normed space $(\mathbb{R},|\cdot|)$ as a Wald space $(\mathbb{R}, \nu, *)$, where

$$
v_{p}=\epsilon_{|p|}=\epsilon_{0}(x-|p|),
$$

for all $p \in \mathbb{R}$. Let $\left(V_{j}, v_{j}, \tau_{j}, \tau_{j}^{*}\right)_{j \in \mathbf{N}}$ be a sequence of Wald spaces where $V_{j}=\mathbb{R}$ for every $j \in \mathbf{N}$. Then their $\Sigma$-product is the pair $(V, v)$, where

$$
V:=\Pi_{j \in \mathrm{N}} V_{j}=\Pi_{j \in \mathrm{N}} \mathbb{R},
$$

and where, for every $p=\left(p_{j}\right) \in V$ one has

$$
v_{p}:=\sum_{j \in \mathbf{N}} \frac{1}{2^{j}} v_{j}\left(p_{j}\right) .
$$

Choose $p=(1,1,0, \ldots, 0, \ldots), q=(0,0,1, \ldots, 1, \ldots)$, and $r=(1,1,1, \ldots, 1, \ldots)$; then $p+q=r$,

$$
v_{p}=\frac{1}{4} \epsilon_{0}+\frac{3}{4} \epsilon_{1}, \quad v_{q}=\frac{3}{4} \epsilon_{0}+\frac{1}{4} \epsilon_{1}, \quad \text { and } \quad v_{r}=\epsilon_{1},
$$

whence one concludes that

$$
v_{p} * v_{q}=\frac{1}{16}\left(3 \epsilon_{2}+10 \epsilon_{1}+3 \epsilon_{0}\right) \text {, }
$$

and, for $0<x<1$,

$$
\left(v_{p} * v_{q}\right)(x)=\frac{3}{16}>0=v_{r}(x),
$$

and the proof is complete.

From the point of view of topology, the following result is essential. 
Theorem 3.3. The strong topology of the $\Sigma$-product $\left(V, v, \tau_{W}, \tau_{W^{*}}\right)$ coincides with the product topology of the sequence of $\operatorname{spaces}\left(V_{j}, v_{j}, \tau_{j}, \tau_{j}^{*}\right)_{j \in \mathrm{N}^{*}}$

Proof. The neighbourhoods of the type

$$
\mathcal{N}_{\theta}(t):=\left\{q \in V: v_{q}(t)>1-t\right\}
$$

form a basis for the topology of the $\Sigma$-product $\left(V, \nu, \tau_{W}, \tau_{W^{*}}\right)$, while the cylinders

$$
\prod_{j=1}^{k} \mathcal{N}_{\theta_{j}}\left(t_{j}\right) \times \prod_{j>k} V_{j}
$$

are a basis for the product topology.

Let a neighbourhood $\mathcal{N}_{\theta}(t)$ be given with $\left.t \in\right] 0,1\left[\right.$ (if $t \geq 1$, then $\mathcal{N}_{\theta}(t)=V$ ). Since there exists $n \in \mathbf{N}$ such that $\sum_{j=1}^{n} \beta_{j}>1-t$, it is possible to define

$$
t^{\prime}:=1-\frac{1-t}{\sum_{j=1}^{n} \beta_{j}}>0
$$

and let $t_{0}:=t \wedge t^{\prime}$. If a point $q$ belongs to the cylinder

$$
U_{n}\left(t_{0}\right):=\prod_{j=1}^{n} \mathcal{N}_{\theta_{j}}\left(t_{0}\right) \times \prod_{j>n} V_{j}
$$

then one has $v_{j}\left(q_{j}\right)\left(t_{0}\right)>1-t_{0}$ for every $j=1,2, \ldots, n$. Now

$$
v_{q}(t) \geq v_{q}\left(t_{0}\right) \geq \sum_{j=1}^{n} \beta_{j} v_{j}\left(q_{j}\right)\left(t_{0}\right)>\sum_{j=1}^{n} \beta_{j}\left(1-t_{0}\right) \geq \sum_{j=1}^{n} \beta_{j}\left(1-t^{\prime}\right)=1-t ;
$$

so that $U_{n}\left(t_{0}\right) \subset \mathcal{N}_{\theta}(t)$.

In the other direction, consider the cylinder

$$
U_{n}\left(t_{1}, t_{2}, \ldots, t_{n}\right):=\prod_{j=1}^{n} \mathcal{N}_{\theta_{j}}\left(t_{j}\right) \times \prod_{j>n} V_{j}
$$

and set

$$
\begin{aligned}
& t:=\min \left\{t_{1}, t_{2}, \ldots, t_{n}\right\}>0 \\
& t^{\prime}:=1-\max \left\{\beta_{j}\left(1-t_{j}\right)+\sum_{\substack{k \in \mathbf{N} \\
k \neq j}} \beta_{k}: j=1,2, \ldots, n\right\} \\
& t_{0}:=t \wedge t^{\prime}
\end{aligned}
$$

Then, if $q$ belongs to the neighbourhood $\mathcal{N}_{\theta}\left(t_{0}\right)$, one has, for every $j=1,2, \ldots, n$,

$$
\begin{aligned}
& v_{q}\left(t_{j}\right) \geq v_{q}\left(t_{0}\right)>1-t_{0} \geq 1-t^{\prime}=\max \left\{\beta_{j}\left(1-t_{j}\right)+\sum_{\substack{k \in \mathbf{N} \\
k \neq j}} \beta_{k}: j=1,2, \ldots, n\right\} \\
& \geq \beta_{j}\left(1-t_{j}\right)+\sum_{\substack{k \in \mathbb{N} \\
k \neq j}} \beta_{k} \geq \beta_{j}\left(1-t_{j}\right)+\sum_{\substack{k \in \mathbb{N} \\
k \neq j}} \beta_{k} \nu_{k}\left(q_{k}\right)\left(t_{j}\right) ;
\end{aligned}
$$

this inequality implies

$$
\beta_{j} v_{j}\left(q_{j}\right)\left(t_{j}\right)>\beta_{j}\left(1-t_{j}\right)
$$

namely $v_{j}\left(q_{j}\right)\left(t_{j}\right)>1-t_{j}$; therefore

$$
\mathcal{N}_{\theta}\left(t_{0}\right) \subset U_{n}\left(t_{1}, t_{2}, \ldots, t_{n}\right) ;
$$

which concludes the proof. 


\section{4. (II)-The $\tau$-product}

By extending Alsina's construction [17] a different definition is possible for the countable product of PN spaces. Before proceeding, it is necessary to recall the definition of the $\tau^{(\infty)}$-product of a sequence $\left(\tau_{n}\right)_{n \in \mathrm{N}}$ of triangle functions. One proceeds inductively by setting, for any sequence $\left(F_{n}\right)_{n \in \mathrm{N}}$ of distribution functions in $\Delta^{+}, \tau^{(1)}:=\tau_{1}$, and, for $n \geq 2$,

$$
\tau^{(n)}\left(F_{1}, \ldots, F_{n}, F_{n+1}\right):=\tau_{n}\left(\tau^{(n-1)}\left(F_{1}, \ldots, F_{n}\right), F_{n+1}\right) .
$$

The sequence $\left\{\tau^{(n)}\left(F_{1}, \ldots, F_{n+1}\right)\right\}$ is decreasing so that its weak limit always exists, although it may be identically be equa to zero; it is denoted by $\tau^{(\infty)}\left\{F_{n}\right\}$,

$$
\tau^{(\infty)}\left\{F_{n}\right\}:=\mathrm{w}-\lim _{n \rightarrow+\infty} \tau^{(n)}\left(F_{1}, \ldots, F_{n+1}\right) .
$$

Definition 4.1. Given a sequence $\left(V_{j}, v_{j}, \tau_{j}, \tau_{j}^{*}\right)_{j \in \mathrm{N}}$ of PN spaces and a continuous triangle function $\tau$, their $\tau$-product is the pair $(V, \nu)$, where $V=\prod_{j \in \mathbf{N}} V_{j}$ and $\nu: V \rightarrow \Delta^{+}$is defined, if $p=\left(p_{n}\right)$ with $p_{n} \in V_{n}$ for every $n \in \mathbf{N}$, by

$$
v_{p}:=\tau^{(\infty)}\left\{v_{n}\left(p_{n}\right)\right\} .
$$

Theorem 4.1. Let $\left(V_{n}, v_{n}, \tau_{n}, \tau_{n}^{*}\right)_{n \in \mathrm{N}}$ be a sequence of PN spaces. Assume that there exist two continuous triangle functions and $\tau^{*}$ such that

(a) for every $n \in \mathbf{N}, \tau \leq \tau_{n} \leq \tau_{n}^{*} \leq \tau^{*}$;

(b) $\tau^{*}$ dominates $\tau, \tau^{*} \gg \tau$.

Then their $\tau$-product $(V, v)$ is a PN space under $\tau$ and $\tau^{*}$.

Proof. One has $v_{\theta}=\tau^{(\infty)}\left\{v_{\theta_{n}}\right\}=\tau^{(\infty)}\left\{\epsilon_{0}, \ldots, \epsilon_{0}, \ldots\right\}=\epsilon_{0}$. Notice that this also proves that $\tau^{(\infty)}$ is not identically equal. to zero. Now assume that $v_{p}=\epsilon_{0}$; then one has $v_{n}\left(p_{n}\right)=\epsilon_{0}$, and hence $p_{n}=\theta_{n}$ for every $n$; therefore $p=\theta$ and (N1) is proved, property (N2) is trivial.

Let $p=\left(p_{n}\right)$ and $q=\left(q_{n}\right)$ be elements of $V$. Then, on account of the continuity of $\tau$, one has

$$
\begin{aligned}
v_{p+q} & =\tau^{(\infty)}\left\{v_{n}\left(p_{n}+q_{n}\right)\right\} \geq \tau^{(\infty)}\left\{\tau_{n}\left(v_{n}\left(p_{n}\right), v_{n}\left(q_{n}\right)\right)\right\} \\
& \geq \tau^{(\infty)}\left\{\tau\left(v_{n}\left(p_{n}\right), v_{n}\left(q_{n}\right)\right)\right\}=\tau\left(\tau^{(\infty)}\left(v_{n}\left(p_{n}\right)\right), \tau^{(\infty)}\left(v_{n}\left(q_{n}\right)\right)\right) \\
& =\tau\left(v_{p}, v_{q}\right),
\end{aligned}
$$

which proves (N3).

For every $\alpha \in[0,1]$, one has, since $\tau^{*}$ is continuous and $\tau^{*}$ dominates $\tau$,

$$
\begin{aligned}
v_{p} & =\tau^{(\infty)}\left\{v_{n}\left(p_{n}\right)\right\} \leq \tau^{(\infty)}\left\{\tau_{n}^{*}\left(v_{n}\left(\alpha p_{n}\right), v_{n}\left((1-\alpha) p_{n}\right)\right)\right\} \\
& \leq \tau^{(\infty)}\left\{\tau^{*}\left(v_{n}\left(\alpha p_{n}\right), v_{n}\left((1-\alpha) p_{n}\right)\right)\right\} \\
& \leq \tau^{*}\left(\tau^{(\infty)}\left\{v_{n}\left(\alpha p_{n}\right)\right\}, \tau^{(\infty)}\left\{v_{n}\left((1-\alpha) p_{n}\right)\right\}\right) \\
& =\tau^{*}\left(v_{\alpha p}, v_{(1-\alpha) p}\right),
\end{aligned}
$$

which proves (N4) and concludes the proof.

In the case of Menger spaces we have a stronger result as a special case of the previous theorem.

Example 4.1. In the previous theorem, if for every $n \in \mathbf{N}, \tau_{n}=\tau_{M}, \tau_{n}^{*}=\tau_{M^{*}}$ and $\tau^{*}=\tau_{M}$, namely, if the spaces $\left(V_{j}, v_{j}, \tau_{j}, \tau_{j}^{*}\right)_{j \in \mathrm{N}}$ are all PN spaces of Menger, then their $\tau_{M}$-product $(V, \nu)$ is also a PN space of Menger denoted by $(V, \nu, M)$

Example 4.2. Under the same hypotheses of Theorem 4.1, assume that for every $n \in \mathbf{N}, \tau_{n}=\tau_{T}, \tau_{n}^{*}=\tau_{M^{*}}$; then the $\tau_{T}$ product $(V, v)$ is a PN space under $\tau_{T}$ and $\Pi_{M}$. Notice that one has $\tau_{M^{*}}^{*} \leq \Pi_{M}$ : in fact, for all $F$ and $G$ in $\Delta^{+}$and $x>0$, one has

$$
\tau_{M^{*}}(F, G)(x)=\tau_{M}(F, G)(x)=\sup \{M(F(u), G(v)) \mid u+v=x\} \leq M(F(x), G(x)),
$$

since for all $u$ and $v$ in $\mathbb{R}$ such that $u+v=x, M(F(u), G(v)) \leq M(F(x), G(x))$ holds. On the other hand $\Pi_{M} \gg \tau$ for every triangle function $\tau$, which establishes the assertion.

However, even if $\left(V_{j}, v_{j}\right)_{j \in \mathrm{N}}$ is a sequence of PN spaces of Menger with respect to the same triangle functions $\tau_{T}$ and $\tau_{T}$ their $\tau_{T}$-product need not be a PN space of Menger with respect to the same triangle functions because $\tau_{T^{*}} \gg \tau_{T}$ is not true. 
In a way similar to that of Theorem 4.1 one proves the following

Theorem 4.2. Under the same assumptions of the previous Theorem, if the mapping $v^{\prime}: V \rightarrow \Delta^{+}$is defined, for every $p=\left(p_{n}\right)$ in $V$, by

$$
v_{p}^{\prime}:=\tau^{*(\infty)}\left\{v_{n}\left(p_{n}\right)\right\},
$$

then the $\tau^{*}$-product $\left(V, v^{\prime}, \tau, \tau^{*}\right)$ of the given sequence of PN spaces is a PN space, called the $\tau^{*}$-product of $\left(V_{j}, v_{j}, \tau_{j}, \tau_{j}^{*}\right)_{j \in N^{*}}$.

Notice that for the probabilistic norms $v$ and $v^{\prime}$ of the $\tau$-and $\tau^{*}$-products, respectively, one has $v \leq v^{\prime}$.

Next, we give the example of a $\tau$-product of a sequence of PN spaces. To this end, recall that an equilateral PN space $(V, v, F)($ see $[7])$ is not a Šerstnev space unless the d.f. $F$ is constant on $\mathbb{R}$.

Example 4.3. Let $\left(\beta_{n}\right)_{n \in \mathbf{N}}$ be a sequence of strictly positive numbers such that $\sum_{n \in \mathbf{N}} \beta_{n}<+\infty$ and let $\left(V_{n}, v_{n}, \Pi_{M}\right)_{n \in \mathbf{N}}$ be a sequence of equilateral PN spaces, where, for all $n \in \mathbf{N}, \nu_{n}=\epsilon_{\beta_{n}}$. The convergence of the series $\sum_{n \in \mathbb{N}} \beta_{n}$ ensures that $\Pi_{M}^{(\infty)}$ is not identically zero. The $\Pi_{M}$-product is again an equilateral PN space. Notice also that, if $\tau$ is a continuous triangle function such that $\tau\left(\epsilon_{a}, \epsilon_{b}\right)=\epsilon_{a+b}$ for all real numbers $a, b \in[0,+\infty]$, then the $\tau$-product $(V, v)$ is a PN space under $\tau$ and $\Pi_{M}$.

It suffices to recall that $\Pi_{M} \gg \tau$ for every triangle function $\tau$.

Theorem 3.3 has only a partial analogue in the case of the $\tau$-product of PN spaces.

Theorem 4.3. The product topology of the sequence $\left(V_{n}, v_{n}, \tau_{n}, \tau_{n}^{*}\right)_{n \in \mathrm{N}}$ of PN spaces is weaker than the strong topology of their $\tau$-product.

Proof. As in the proof of Theorem 3.3 let $U_{n}\left(t_{1}, t_{2}, \ldots, t_{n}\right)$ be the same neighbourhood as in (3.3) and define $t:=$ $\min \left\{t_{1}, \ldots, t_{n}\right\}>0$. Let $q=\left(q_{n}\right)$ belong to $N_{\theta}(t)$; then, one has, for all $j \in\{1,2, \ldots, n\}$,

$$
1-t_{j} \leq 1-t<v_{q}(t) \leq v_{j}\left(q_{j}\right)(t) \leq v_{j}\left(q_{j}\right)\left(t_{j}\right) \text {, }
$$

so that $N_{\theta}(t) \subset \prod_{j \in \mathrm{N}} N_{\theta_{j}}\left(t_{j}\right) \subset U_{n}\left(t_{1}, t_{2}, \ldots, t_{n}\right)$.

Considerations analogous to those presented in [17] could be made in order to explain (i) why the two topologies that are the object of Theorem 4.3 in general cannot coincide and (ii) the different probabilistic meaning, in the topologies of $\sigma$ - and $\tau$-products, of the statement "a point $p=\left(p_{n}\right)$ belongs to the neighbourhood $\mathbf{N}_{\theta}(t)$ ". Since they would just be a rephrasing of those in Alsina's paper, they will not be repeated here.

\section{Final considerations}

In [18] Alsina and Schweizer were successful in constructing the countable product of Probabilistic Metric(= PM) spaces in such a way that the following natural, and, hence, desirable, properties of the product space hold

(a) if all the PM spaces in the sequence $\left(S_{n}, \mathcal{F}_{n}, \tau\right)_{n \in \mathrm{N}}$ share the same triangle function $\tau$, then the product space $(S, \mathcal{F})$ is a PM under the same triangle function $\tau$;

(b) the strong topology of the product space coincides with the product topology.

One naturally wonders whether the construction given in [18] may be modified so as to extend it to the case of the countable product of PN, rather than PM, spaces. It will be shown below that the answer to this question must be negative. This is a consequence of the fact that two triangle functions, $\tau$ and $\tau^{*}$, rather than only one, appear in the definition of a PN space.

Let $\left(V_{j}, \nu_{j}, \tau, \tau^{*}\right)_{j \in \mathrm{N}}$ be a sequence of PN spaces under the same two continuous triangle functions $\tau$ and $\tau^{*}$.

The same concepts will be used as in [18]. As in that paper, let $\left(\beta_{j}\right)_{j \in \mathbf{N}}$ be a sequence of strictly positive numbers such that the series $\sum_{j \in \mathbf{N}} \beta_{j}$ converges. For every $j \in \mathbf{N}$, let $\varphi_{j}$ belong to $M_{\beta_{j}}$, the set of strictly increasing functions from $\left[0, \beta_{j}\right]$ onto $[0,+\infty]$. The functions $\varphi_{j}$ 's are requested to be $\tau$-superadditive, viz., for all $F$ and $G$ in $\Delta^{+}$and for every $j \in \mathbf{N}$,

$$
\tau(F, G) \circ \varphi_{j} \geq \tau\left(F \circ \varphi_{j}, G \circ \varphi_{j}\right) .
$$

Define, for every $j \in \mathbf{N}, \widetilde{v}\left(p_{j}\right):=v\left(p_{j}\right) \circ \varphi_{j}$ and, for $p=\left\{p_{j}: j \in \mathbf{N}\right\}$ in $\prod_{j \in \mathbf{N}} V_{j}$,

$$
\varphi_{p}:=\tau^{\infty}\left\{\tilde{\nu}\left(p_{j}\right)\right\} .
$$

Then again, with the same proof as in [18], one shows that $v_{p}$ belongs to $\mathcal{D}^{+}$and that the probabilistic norm thus defined satisfies property (N3) of a PN space. The problems arise with property (N4). In fact, in order to satisfy this latter property one has to require that each $\varphi_{j}$ be $\tau^{*}$-subadditive, namely, for all $F$ and $G$ in $\Delta^{+}$and for every $j \in \mathbf{N}$,

$$
\tau^{*}(F, G) \circ \varphi_{j} \leq \tau^{*}\left(F \circ \varphi_{j}, G \circ \varphi_{j}\right)
$$


[2] A.N. Šerstnev, On the notion of a random normed space, Dokl. Akad. Nauk. SSSR 149 (2) 280-283, Soviet Math. Doklady, 4 (1963) 388-390.

[3] A.N. Šerstnev, Best approximation problems in random normed spaces, Dokl. Akad. Nauk. SSSR 149 (3) (1963) 539-542.

[4] A.N. Šerstnev, On a probabilistic generalization of a metric spaces, Kazan Gos. Univ. Učen. Zap. 124 (1964) 3-11.

[5] C. Alsina, B. Schweizer, A. Sklar, On the definition of a probabilistic normed space, Aequationes Math. 46 (1993) 91-98.

[6] B. Lafuerza Guillén, J.A. Rodríguez Lallena, C. Sempi, Completion of probabilistic Normed spaces, Internat. J. Math. Math. Sci. 18 (1995) 649-652.

[7] B. Lafuerza Guillén, J.A. Rodríguez Lallena, C. Sempi, Some classes of probabilistic normed spaces, Rend. Mat. 7 (17) (1997) $237-252$.

[8] B. Lafuerza Guillén, J.A. Rodríguez Lallena, C. Sempi, Probabilistic norms for linear operators, J. Math. Anal. Appl. 220 (1998) $462-476$.

[9] B. Lafuerza Guillén, J.A. Rodríguez Lallena, C. Sempi, A study of boundedness in probabilistic normed spaces, J. Math. Anal. Appl. 232 (1999) 183-196.

[10] C. Sempi, A short and partial history of probabilistic normed spaces, Mediterr. J. Math. 3 (2006) 283-300. doi: 10.1007/s00009-006-0078-6.

[11] B. Schweizer, A. Sklar, Probabilistic Metric Spaces, 2nd ed., Elsevier, New York, 1983, Dover, Mineola, NY, 2005.

[12] E.P. Klement, R. Mesiar, E. Pap, Triangular Norms, Kluwer, Dordrecht, 2000.

[13] C. Alsina, M.J. Frank, B. Schweizer, Associative Functions. Triangular Norms and Copulas, World Scientific, Singapore, 2006.

[14] S. Saminger-Platz, C. Sempi, A primer on triangle functions. I, Aequationes Math. 76 (2008) 201-240.

[15] S. Saminger-Platz, C. Sempi, A primer on triangle functions. II, Aequationes Math. (in press).

[16] B. Lafuerza Guillén, Finite products of probabilistic normed spaces, Rad. Mat. 13 (2004) 111-117.

[17] C. Alsina, On countable products and algebraic convexification of probabilistic metric spaces, Pacific J. Math. 76 (1978) 291-300.

[18] C. Alsina, B. Schweizer, The countable products of probabilistic metric spaces, Houston J. Math. 9 (1983) 303-310.

[19] W. Jarczyk, J. Matkowski, On Mulholland's inequality. Proc. Amer. Math. Soc. 130 (2002) 3243-3247.

[20] H. Sherwood, Characterizing dominates in a family of triangular norms, Aequationes Math. 27 (1984) 255-273.

[21] R.M. Tardiff, On a generalized Minkowski inequality and its relation to dominates for $t$-norms, Aequationes Math. 27 (1984) 308-316.

[22] P. Sarkoci, Dominance is not transitive on continuous triangular norms, Aequationes Math. (in press).

I23l R M Tardiff Tonologies for nrobabilistic metric snaces Ph D Dissertation University of Massachusetts Amherst. 1975 